(REVIEW ARTICLE)

\title{
Oral cancer: etiology, precancerous lesions and treatment: A literature review
}

Joe Javier Lalangui Matamoros ${ }^{1,}{ }^{*}$, Doménica Alejandra Solano Maldonado ${ }^{1}$, Andrea Elizabeth Quito Zhinín ${ }^{1}$ and Fernando Mauricio Villalta Mendoza ${ }^{2}$

${ }^{1}$ Student of Faculty of Dentistry, University of Cuenca.

${ }^{2}$ Bachelor in General Dentistry Faculty of Dentistry, University of Cuenca.

Publication history: Received on 19 November 2020; revised on 27 November 2020; accepted on 29 November 2020

Article DOI: https://doi.org/10.30574/wjarr.2020.8.2.0446

\begin{abstract}
Introduction: Oral cancer is one of the most common malign neoplasia around the world. However, despite current diagnosis tools, a high morbidity and mortality rate is present due to a late diagnosis and a lack of population consciousness about tobacco, alcohol, and other substances consumption. Objective: The current review aims to provide updated information about precancerous lesions, etiology, diagnosis, and treatment of oral cancer. Methodology: A bibliographic research on peer review articles were done in five digital repositories considering publications from 2015 up to date. Conclusion: Oral cancer is a frequent pathology with severe consequences for its affected patients. A wide knowledge on pre-malign lesions, risk factors, and early diagnosis is key to prevent the presence and development of this pathology.
\end{abstract}

Keywords: Oral cancer; Carcinogenesis; Etiology; Diagnosis; Treatment; Risk factors; Precancerous lesion

\section{Introduction}

Oral cancer is one of the most incident pathologies worldwide, representing the eleventh most common malign neoplasia, and the second death cause after cardiovascular diseases [1]. Besides the significant advances in oral cancer research and diagnosis, it has remained constant in population due to alcohol and tobacco consumption as well as low dental visits frequency. This incurs in late diagnosis with increasing morbidity and mortality rates [2-4]. The current review aim is to provide updated information on oral cancer remarkable aspects, its etiology, precancerous lesions, diagnosis, and treatment, highlighting the early diagnosis value.

\section{Material and methods}

We made a detailed literature research in the digital repositories: Pubmed, Science Direct, Cochrane, Scopus and Springerlink, using the following keywords: "Oral cancer", "Carcinogenesis", "Etiology", "Diagnosis", "Treatment", "Risk factor" and "Precancerous lesion" with Boolean operators Or - And.

\subsection{Inclusion criteria}

Peer review articles published in the last five years with complete text availability.

\subsection{Exclusion criteria}

- $\quad$ Studies with no clear age groups.

\footnotetext{
${ }^{*}$ Corresponding author: Joe Javier Lalangui Matamoros

Student of Faculty of Dentistry, University of Cuenca. 
- $\quad$ Studies with unavailable oral cancer data or its aggrupation with other lesions data, which does not allow its extraction.

- $\quad$ Studies with no clinic exam for oral cancer diagnosis and no confirmation through histopathological analysis.

- $\quad$ Studies based on case reports, protocols, brief communications, personal opinions, or letters.

Finally, complete text from 24 publications were selected and evaluated

\section{Results}

\subsection{Definition}

Oral cancer is a lip or oral cavity malign neoplasia [2-5], representing one of the most common head and neck cancer that includes a wide variety of malign tumors such as scaly cells carcinomas, malign tumors in the lower salivary glands, lymphomas, sarcomas, melanomas, odontogenetic carcinomas, metastatic diseases, among others [6].

Preventive actions are fundamental for the pathology, considering treatment high costs and low affordability [1,7]. Oral cancer clinical manifestations and treatment effects can present negative consequences in patient life quality, mainly for capacities decrease in talking, eating activities, along with face aesthetic alterations, sensorial damage, and chronic pain [8].

\subsection{Carcinogenesis}

The classic carcinogenesis model includes three phases. In the initiation (1st), carcinogenic biological elements introduce oncoproteins in the target cell, altering its genetics code, activating protooncogenes, and inactivating tumors suppressor genes. During the promotion (2nd) tissue growth and cancer can develop through overexpression of receptors and growth factors, cytosines, outer cell-matrix destruction, angiogenesis, etc. Finally, malign cells invade local or distal tissues in the progression (3rd), while molecular alterations keep on [9].

\subsection{Epidemiology}

The percentage of cancer prevalence by region according to the World Health Organization (WHO) is distributed in Southwest Asia (42\%), Europe (18,5\%), and Latin America and Caribe (13.2\%) with a 50\% mortality rate [10]. According to gender, oral cancer can be twice or thrice times more common in male individuals [3,11]. 40\% of head and neck cancers have a place in the oral cavity, $15 \%$ in the pharynx, and $25 \%$ in the larynx with tumors in other places like salivary glands and thyroids [12]. Location of cancer inside the oral cavity is distributed in oral mucosa (32\%), tongue (22\%), lower lip (11\%), palate (11\%), vestibular (8\%), alveolus (5\%), mouth floor (5\%), and gum (3\%) [1]. 90\% of malign neoplasia represents the scaly carcinoma cells (CCE) [1,3].

\subsection{Etiology}

As a multifactor disease, it presents a relation of 75\% with individuals' lifestyle. Its etiology divides into genetic and epigenetic factors [1].

\subsubsection{Genetic factors}

Nowadays discovery and comprehension of oral cancer genetic alterations increased significantly due to genetic instability manifestations on solid tumors [13]. Application of high-performance multiple techniques for genomic variations research resulted in two main observations:

- $\quad$ Same-origin tumors vary considerably regarding to its genomic variations [1].

- $\quad$ Tumors present similar genomic variation patterns with different origins [1].

\subsubsection{Epigenetic factors}

Tobacco

Tobacco components demonstrated to cause cancer at smoking or chewing. The pathology development likelihood is five times higher in people that smoke, considering that 300 identified saliva-soluble carcinogenic components are present in tobacco smoke $[14,16]$. 
Alcohol

Alcoholic drinks considered carcinogenic can cause tumors in oral cavity, larynx, esophagus and liver. Alcohol increases pre-carcinogens activation and behave as a solvent for enter of harmful carcinogens in body cells. Oral cancer detection is complex in its first phases due to the symptom's absence. Therefore, early diagnosis is imperative to increase patient survival [1].

Diet and nutrition

Several epidemiological and laboratory studies link diet and nutrition with cancer development. A deficient diet in antioxidants, vitamins, minerals, and oligo-elements predisposes to develop oral and pharynx cancer [4].

Certain food groups associates with a higher mouth cancer development, such as: processed meat, cakes and desserts, butter, eggs, soups, red meat, salt meat, cheese, legumes, pasta or rice, and cornbread [17].

Virus and simple herpes

Herpes simplex virus (HSV) is a risk factor in the oral scam cells carcinoma creation. The transformation to a malign phenotype makes the virus to behave as a mutagen and isolates an area of the viral genome, increasing the mutation frequency in cultured cells [1].

Precancerous lesions

An oral precancerous lesion defines like any oral mucus lesion or condition with potential for malign transformation. The most common series of oral lesions includes: leukoplakia, erythroplakia, and erythroleukoplakia $[1,18]$.

Oral leukoplakia is the most common oral precancer, representing $85 \%$ of these pathologies $[18,19]$. It presents as a spot or white patch that is not clinically of pathologically characterized as another disease. It mainly affects patients older than 40 years and its malign transformation takes five years on average [18]. About 70\% of oral leucoplakias founds in vermilion lip, oral mucus, and gums with $90 \%$ of them showing dysplasia or carcinoma [19].

Oral erythroplakia defines as a red patch that cannot clinically or pathologically diagnosticate like any other affection. It characterizes by an altered mucus that appears like a macule or well delimited erythematous patch with a soft and velvety texture. Most of the time this pathology shows a significant epithelial dysplasia, in situ or invasive scaly cell carcinoma. Oral erythroplakia can be independent or associated with oral leukoplakia, denominating erythroleukoplakia, a red and white colored lesion with patterns of leukoplakia that frequently shows advanced dysplasia through biopsy $[18,19]$.

\subsection{Diagnosis}

People with an affected site alteration last four to six months to find professional help (8 to 24 months in lower socioeconomic groups). Minimum pain presents during the initial growth phase, which explains the delay in finding professional attention [1]. An analysis of precancerous lesions main characteristics is necessary to determine a leukoplakic, erythroplakia, or erythroleukoplakic lesion. Health professional suspect must be confirmed through a biopsy, observing whether exists abnormal changes in cells compatible with a malign disease diagnosis [20].

The more important histopathological characteristics to consider in biopsy are a dysplastic epithelial surface, characterized by: hyperchromatic nuclei, increased size cells with polarity lack, dyskeratosis, augment of miotic activity, and keratin pearls.

This abnormal epitheliumcan irregularly invade the basal membrane and subepithelial connective tissue [1]

\subsection{Treatment}

Besides type, stage, and location of oral cancer, systemic health and patient nutrition stage influence in therapeutics particularly. First-line treatment for oral cancer is surgery, followed by chemoradiotherapy [21]. 


\subsubsection{Surgery}

Surgery keeps as the first oral cancer treatment modality that divides into "resective" and "reconstructive" compounds. Resection surgery includes primary tumor extraction and cervical ganglion management, while reconstructive surgery essentially implies resection morbidity minimization (e.g.: tissue replacement in talking, swallowing, and mastication effects minimization) [21]. Surgery must be done with five millimeters security margin as the minimum standard accepted for $21 \%$ relapse risk reduction [23].

Most of surgeries involving big tumors resection disfigure and produce undesired effects on patients [24], deteriorating their life quality due to drastic alterations in chewing, swallowing, talking, or facial aesthetic [21]. When surgery is not sufficient to eradicate locally advanced cancer it is commonly followed by radiotherapy [24].

\subsubsection{Radiotherapy}

Post-operatory radiotherapy indicated in oral cavity cancer implies ionizing radiation use to destroy or damage cancerous cells [22]. However, the treatment decision depends to a large extent because of the final histopathological result and its stage. Tumor pathological staging is of vital importance, considering whether existed positive lymphatic ganglions and surgical margin stage.

\subsubsection{Chemotherapy}

Palliative chemotherapy is the pillar treatment for relapsing or metastatic tumors that are not adequate for rescue surgery or re-irradiation, it helps to preserve tissues as well as patient survival depleting contributory radiotherapy quantity [23].

\subsubsection{Monitoring}

Monitoring must realize at least two times a year and adapt regarding necessities. Any traumatic dental procedure after radiotherapy must make under antibiotics coverture [12].

Recurrences occur in up to $2 / 3$ of oral cancer patients, late detected due to extensive treatments that difficult their identification [23]

\section{Conclusion}

Precancerous lesions are the beginning of oral cancer, with professional responsibility to make a correct diagnosis, correctly identifying such lesions or patient predisposition to suffer it, make an exhaustive anamnesis and clinic evaluation to recognize present signs and factors that influence on them as tobacco use, alcohol consumption, malnutrition, among others. Furthermore, professional decisions after diagnosis plays an important role because of treatment determines patient life's quality.

Furthermore, oral cancer detected in early stages have a better prediction and preventable with patient and dentist intervention, avoiding harmful habits, and making a correct diagnostic evaluation with good treatment plans.

\section{Compliance with ethical standards}

\section{Acknowledgments}

Acknowledgments to Dr. Marina Abril for her review and selfless help in the manuscript.

\section{Disclosure of conflict of interest}

All the authors state no conflict of interest for publishing this original manuscript

\section{References}

[1] S D'souza, V. Addepalli. Preventive measures in oral cancer: An overview. Biomedicine \& Pharmacotherapy. 2018; 107: 72-80.

[2] Y Ghantous, A Elnaaj. Global incidence and risk factors of oral cancer. Harefuah. 2017; 156(10): 645-9. 
[3] C Rivera. Essentials of oral cancer. International journal of clinical and experimental pathology. 2015; 8(9): 11884.

[4] S Manoharan, S Karthikeyan, MM Essa, A Manimaran, R Selvasundram. An overview of oral carcinogenesis. International Journal of Nutrition, Pharmacology, Neurological Diseases. 2016; 6(2): 51.

[5] AD Shrestha, P Vedsted, P Kallestrup, D Neupane. Prevalence and incidence of oral cancer in low-and middleincome countries: A scoping review. European Journal of Cancer Care. 2020; 29(2): e13207.

[6] CGB Alves, NS Treister, ACP Ribeiro, TB Brandão, JO Tonaki, MA Lopes, et al. Strategies for communicating oral and oropharyngeal cancer diagnosis: why talk about it? Oral surgery, oral medicine, oral pathology and oral radiology. 2019.

[7] P Ramos-Garcia, MdM Roca-Rodriguez, M Aguilar-Diosdado, MA Gonzalez-Moles. Diabetes mellitus and oral cancer/oral potentially malignant disorders: A systematic review and meta-analysis. Oral Diseases. 2020.

[8] JA Valdez, MT Brennan. Impact of oral cancer on quality of life. Oral Cancer, An Issue of Dental Clinics of North America, E-Book. 2017; 62(1): 143.

[9] I Lafuente Ibáñez de Mendoza, X. Maritxalar Mendia , A.M. García de la Fuente, A.G. Quindós, J.M. Aguirre Urizar. Role of Porphyromonas gingivalis in oral squamous cell carcinoma development: A systematic review. Journal of periodontal research. 2020; 55(1): 13-22.

[10] F Bray, J Ferlay, I Soerjomataram, RL Siegel, LA Torre, A Jemal. Global cancer statistics GLOBOCAN estimates of incidence and mortality worldwide for 36 cancers in 185 countries. CA: a cancer journal for clinicians. 2018; 68(6): 394-424.

[11] JM García-Martín, P Varela-Centelles, M González, JM Seoane-Romero, J Seoane, MJ García-Pola. Epidemiology of oral cancer. Oral Cancer Detection: Springer. 2019; 81-93.

[12] A Villa, SO Akintoye. Dental management of patients who have undergone oral cancer therapy. Dental Clinics. 2018; 62(1): 131-42.

[13] GS Sarode, SC Sarode, N Maniyar, R Anand, S Patil. Oral cancer databases: A comprehensive review. Journal of oral pathology \& medicine. 2018; 47(6): 547-56.

[14] P Chaturvedi, A Singh, CY Chien, S Warnakulasuriya. Tobacco related oral cancer. BMJ. $2019 ; 365$.

[15] S Tomar, SS Hecht, I Jaspers, R Gregory, I Stepanov. Oral health effects of combusted and smokeless tobacco products. Advances in dental research. 2019; 30(1): 4-10.

[16] M Pemberton. Oral cancer and tobacco: developments in harm reduction. British dental journal. 2018; 225(9): 822.

[17] M Kumar, R Nanavati, TG Modi, C Dobariya. Oral cancer: Etiology and risk factors: A review. Journal of cancer research and therapeutics. 2016; 12(2): 458.

[18] M Awadallah, M Idle, K Patel, D Kademani. Management update of potentially premalignant oral epithelial lesions. Oral surgery, oral medicine, oral pathology and oral radiology. 2018; 125(6): 628-36.

[19] OS Gileva, TV Libik, KV Danilov. Oral precancerous lesions: Problems of early detection and oral cancer prevention. AIP Conference Proceedings; AIP Publishing LLC. 2016.

[20] JB Epstein, C Miaskowski. Oral Pain in the Cancer Patient. JNCI Monographs. 2019; (53): lgz003.

[21] IP Ribeiro, L Barroso, F Marques, JB Melo, IM Carreira. Early detection and personalized treatment in oral cancer: the impact of omics approaches. Molecular Cytogenetics. 2016; 9(1): 1-7.

[22] T Wong, D Wiesenfeld. Oral cancer. Australian Dental Journal. 2018; 63: S91-S9.

[23] AKD'Cruz, R Vaish, H Dhar. Oral cancers: Current status. Oral oncology. 2018; 87: 64-9.

[24] N D'Silva, J Gutkind. Oral cancer: Integration of studies for diagnostic and therapeutic precision. Advances in dental research. 2019; 30(2): 45-9. 